hep-th/0101129

HUB-EP-01/03

\title{
Fibrewise T-Duality for D-Branes on Elliptic Calabi-Yau
}

\author{
Björn Andreas ${ }^{1}$, Gottfried Curio ${ }^{2}$, Daniel Hernández Ruipérez ${ }^{3}$ and Shing-Tung Yau ${ }^{1}$ \\ ${ }^{1}$ Department of Mathematics, Harvard University, Cambridge, MA 02138, USA \\ ${ }^{2}$ Humboldt-Universität zu Berlin, Institut für Physik, D-10115 Berlin, Germany \\ ${ }^{3}$ Departamento de Matemáticas, Universidad de Salamanca, 37008, Salamanca, Spain
}

Fibrewise T-duality (Fourier-Mukai transform) for D-branes on an elliptic Calabi-Yau $X$ is shown to require naturally an appropriate twisting of the operation respectively a twisted charge. The fibrewise T-duality is furthermore expressed through known monodromies in the context of Kontsevich's interpretation of mirror symmetry. 
This paper constitutes a shortened and simplified version of [1] (where details and references are given) intended to present the essential results to an audience of physicists.

The last years saw an intense study on BPS D-branes, with their associated bundles, in type II string theories on a Calabi-Yau manifold. For the class of elliptic Calabi-Yau's not only the description of bundles on the whole Calabi-Yau, related to D6-branes, can be made explicit via spectral covers (cf. [2]), but it allows also for a version of the FourierMukai (FM) transform of a bundle $V$ on non-toroidal spaces which in contrast to earlier transforms in such cases (say on $K 3$ ) sticks completely to the idea of using the duality on a torus by building a fibrewise FM transform. This 'fibrewise T-duality' operates on the spectrum of D-branes and has also a close connection with the spectral cover construction.

We compute the cohomological invariants of a bundle and its fibrewise dual and show how the adiabatic character 1 of the operation is confirmed only by using an appropriately twisted charge. Alternatively a version of Mukai's f-map, using the usual Mukai vector as charge but a slightly twisted operation, has to be used.

Also, in a version of the mirror correspondence, which brings supersymmetric Dbranes on both sides into the play, bundles, or better sheaves (think of them as bundles supported on holomorphic subvarieties), on a Calabi-Yau $X$ in type IIA are related with special Lagrangian submanifolds (with an $U(1)$ bundle over them) in the mirror CalabiYau $Y$ with cohomological invariants relating $H^{\text {even }}(X)$ and $H^{3}(Y)$. Furthermore, the cohomological effect of a line bundle twist (or more general operations related to FM transforms) is related with a type IIB monodromy resp. (using the mirror identification) with a type IIA monodromy (around the locus in moduli space where the divisor vanishes). The fibrewise FM transform is given its place in Kontsevich's general association of FM transforms with monodromies.

Consider an $S U(n)$ bundle $V$ of $c_{1}(V)=0$ over an elliptically fibered $X$ or equivalently $n$ D6-branes wrapped over $X$ with induced D2-and D0-brane charges (D2i-charges meaning here for now just $c h_{i}(V)$ ). The T-duality on the $T^{2}$ fibre maps (subscripts indicate that fibre $F$ or base $B$ is contained (resp. contains) the wrapped world-volume)

$$
\begin{gathered}
D 6 \rightarrow \tilde{D} 4_{B} \\
D 4_{B} \rightarrow \tilde{D} 6 \quad, \quad D 4_{F} \rightarrow \tilde{D} 2_{B} \\
D 2_{B} \rightarrow \tilde{D} 4_{\tilde{F}}, \quad D 2_{F} \rightarrow \tilde{D} 0 \\
D 0 \rightarrow \tilde{D} 2_{\tilde{F}}
\end{gathered}
$$

The operation of fibrewise T-duality can be described on the cohomological data representing $V$ and its FM dual $\tilde{V}$, i.e. we will mirror the mentioned D-brane relations as a map

1 I.e., decomposing the cohomology into base and fibre parts, the operation of fibrewise duality takes the form of an adiabatic extension of the same operation on the (cohomology of a) $T^{2}$, fulfilling the expectations from the interpretation as T-duality on D-branes. 
between $\operatorname{ch}(V)$ and $\operatorname{ch}(\tilde{V})$ (this will be made precise). Assume the following decomposition

$$
\left(\begin{array}{ccc}
H^{0}(X) & = & \mathbf{C} \\
H^{2}(X) & = & \mathbf{C} \sigma \oplus H^{2}(B) \\
H^{4}(X) & = & H^{2}(B) \sigma \oplus \mathbf{C} \\
H^{6}(X) & = & \mathbf{C}
\end{array}\right)
$$

Then essentially the six entries of the Chern character vector in our decomposition are pairwise interchanged and the transformation has the block-diagonal form

$$
Q=\left(\begin{array}{cccccc}
0 & * & & & & \\
* & 0 & & & & \\
& & 0 & * & & \\
& & * & 0 & & \\
& & & & 0 & * \\
& & & & * & 0
\end{array}\right) \tilde{Q}
$$

So the cohomological effect of the fibrewise FM transform (T-duality) is given by adiabatic extension of the effect of T-duality on the fibre where the matrix is [3]

$$
A=\left(\begin{array}{cc}
0 & 1 \\
-1 & 0
\end{array}\right)
$$

So one would like to see as actual form of the transformation matrix

$$
Q=\left(\begin{array}{cccccc}
0 & 1 & 0 & 0 & 0 & 0 \\
-1 & 0 & 0 & 0 & 0 & 0 \\
0 & 0 & 0 & 1 & 0 & 0 \\
0 & 0 & -1 & 0 & 0 & 0 \\
0 & 0 & 0 & 0 & 0 & 1 \\
0 & 0 & 0 & 0 & -1 & 0
\end{array}\right) \tilde{Q}
$$

thereby confirming the 'adiabatic' interpretation of the Fourier-Mukai transform as (with entries now consisting of $3 \times 3$ blocks corresponding to $\left.H^{*}(X)=\sigma H^{*}(B) \oplus \pi^{*} H^{*}(B)\right)$

$$
\left(\begin{array}{cc}
\mathbf{0}_{3} & \mathbf{1}_{3} \\
-\mathbf{1}_{3} & \mathbf{0}_{3}
\end{array}\right)
$$

\section{The Fourier-Mukai transform}

The spectral cover construction日 describes the bundle $V$ from a line bundle $L$ on the spectral cover surface $C$. For the description of the FM transform we will instead of

2 An $S U(n)$ bundle $V$ on $X$ of $c_{1}(V)=0$ decomposes on the typical fibre $E$ (where $V$ is assumed to be semistable) as a sum of line bundles of degree zero each of which corresponds (a reference point $p$ chosen) to a point $Q_{i}$ on $E$. When globalized this leads to a hypersurface $i: C \hookrightarrow X$, a ramified $n$-fold 'spectral cover' of $B$, whose equation can still be twisted so that $C=n \sigma+\eta$ in cohomology. As fibrewise $V=p_{*} \mathcal{P}$ and a twist by a line bundle $L$ over $C$ leaves the fibrewise isomorphism class unchanged this generalizes to $V=p_{*}\left(p_{C}^{*} L \otimes \mathcal{P}\right)$ where $p$ and $p_{C}$ are the projections on the first and second factor of $X \times_{B} C$. The condition $c_{1}(V)=0$ translates to the fixing $\left.\pi_{*}\left(c_{1}(L)\right)=-\pi_{*}\left(c_{1}(C)-c_{1}\right) / 2\right)$ of $c_{1}(L)$ in $H^{1,1}(C)$ up to a class in ker $\pi_{*}: H^{1,1}(C) \rightarrow H^{1,1}(B)$. 
working on $X \times_{B} C$ work on $X \times_{B} \tilde{X}$

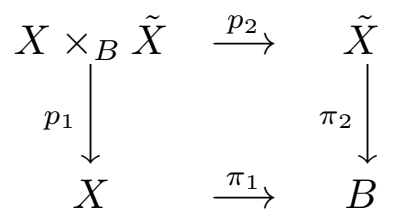

where $\tilde{X}$ is the compactified relative Jacobian of $X . \tilde{X}$ parameterizes torsion-free rank 1 and degree zero sheaves of the fibres of $X \rightarrow B$ and is actually isomorphic with $X$ (see [4] or [5]). We will identify $\tilde{X}$ and $X . V$ is constructed from the Poincaré sheaf $\mathcal{P}$ via

$$
V=p_{1 *}\left(p_{2}^{*}\left(i_{*} L\right) \otimes \mathcal{P}\right)
$$

with $\mathcal{P}=\mathcal{O}\left(\Delta-\sigma \times \tilde{X}-X \times \tilde{\sigma}-c_{1}(B)\right)$ normalized to be trivial along $\sigma \times \tilde{X}$ and $X \times \tilde{\sigma}$.

Let us determine the FM-transform. For this we make use of the fact that the representation of $V$ by the $(C, L)$ data already looks in itself like a FM transform. So the FM transform of $V$ is practically the double transform of $i_{*} L$. But the inverse transform of FM is not precisely FM itself, but rather a slightly twisted version of the first transform, as we will recall. Only this twisted version brings us back from $V$ to $i_{*} L$, so if we start on $V$, making the original FM transform we get $i_{*} L$ times the inverse twist.

So for $V=p_{1 *}\left(p_{2}^{*}\left(i_{*} L\right) \otimes \mathcal{P}\right)$ the 'fibrewise dual' is given in spectral cover data by

$$
\tilde{V}=R^{1} p_{2 *}\left(p_{1}^{*}(V) \otimes \mathcal{P}^{*}\right)=i_{*} L \otimes \pi_{2}^{*} K_{B}
$$

One wants to show that there exists a matrix $M$ in the block diagonal form (5) relating $V$ to $\tilde{V}$ or $V$ to $i_{*} L$ (the latter option is simply the inverse process as $V$ is the dual of $i_{*} L$ ).

Before going on let us recall the FM transform for elliptic fibrations (cf. [4], [3], [5]). We define the Fourier-Mukai functors $S^{i}, i=0,1$ by associating with every sheaf $V$ on $X$ the sheaf $S^{i}(F)$ on $X$ ( $X$ and $\tilde{X}$ are identified; $\mathcal{P}$ the Poincare sheaf on the fibre product)

$$
S^{i}(V)=R^{i} p_{1 *}\left(p_{2}^{*}(V) \otimes \mathcal{P}\right)
$$

It can be also described as (cf. [2] ) $\mathcal{P}=\mathcal{I}^{*} \otimes p_{1}^{*} \mathcal{O}(-\sigma) \otimes p_{2}^{*} \mathcal{O}(-\sigma) \otimes q^{*} K_{B}$ with $q=$ $\pi \cdot p_{1}=\pi \cdot p_{2}$ and $\mathcal{I}=\mathcal{O}(\Delta)^{*}$ the ideal sheaf of the diagonal immersion $\delta: X \rightarrow X \times_{B} X$.

We can also define the inverse Fourier-Mukai functors $\hat{S}^{i}, i=0,1$

$$
\hat{S}^{i}(V)=R^{i} p_{2 *}\left(p_{1}^{*}(V) \otimes \mathcal{P}^{*} \otimes q^{*} K_{B}^{-1}\right)
$$

The relationship between these functors is stated best if we consider the associated functors between the derived categories of complexes of coherent sheaves bounded from above.

$$
\begin{array}{ll}
S: D^{-}(X) \rightarrow D^{-}(X) ; \quad S(\mathcal{G})=R p_{1 *}\left(p_{2}^{*}(\mathcal{G}) \otimes \mathcal{P}\right) \\
\hat{S}: D^{-}(X) \rightarrow D^{-}(X) ; \quad \hat{S}(\mathcal{G})=R p_{2 *}\left(p_{1}^{*}(\mathcal{G}) \otimes \mathcal{P}^{*} \otimes q^{*} K_{B}^{-1}\right)
\end{array}
$$


Note that one obtains an invertibility result

$$
S(\hat{S}(\mathcal{G}))=\mathcal{G}[-1], \quad \hat{S}(S(\mathcal{G}))=\mathcal{G}[-1]
$$

The K3 case

Consider first the two-dimensional case of $X=K 3$ and assume the decomposition

$$
\left(\begin{array}{lcc}
H^{0}(X) & = & \mathbf{C} \\
H^{2}(X) & = & \mathbf{C} \sigma \oplus \mathbf{C} \\
H^{4}(X) & = & \mathbf{C}
\end{array}\right)
$$

The class of the spectral curve $C$ on which $i_{*} L$ is supported is $C=n \sigma+k F$.

The Chern characters of $i_{*} L$ can be obtained using Grothendieck-Riemann-Roch for the embedding $i: C \rightarrow \tilde{X}$, giving with $\operatorname{ch}\left(i_{*} L\right) T d(\tilde{X})=i_{*}(\operatorname{ch}(L) T d(C))$ that

$$
\operatorname{ch}\left(i_{*} L\right)=(0, C, n)
$$

further $\operatorname{ch}(V)$ ( $V$ is the only FM transform of $\left.i_{*} L\right)$ is given by

$$
\operatorname{ch}(V)=(n, 0,-k)
$$

Let us introduce 3 the new functor $T(\cdot)=S(\cdot) \otimes \pi^{*} K_{B}^{-1 / 2}=S(\cdot) \otimes \mathcal{O}(F)$ (and similarly $\left.T^{i}(\cdot)=S^{i}(\cdot) \otimes \pi^{*} K_{B}^{-1 / 2}=S^{i}(\cdot) \otimes \mathcal{O}(F)\right)$ so that $T^{0}\left(i_{*} L\right)=V \otimes \mathcal{O}(F)$. We get

$$
\operatorname{ch}\left(T^{0}\left(i_{*} L\right)\right)=\operatorname{ch}(V)(1+F)=M \cdot \operatorname{ch}\left(i_{*} L\right)
$$

where we reach the matrix we wanted

$$
M=\left(\begin{array}{cccc}
0 & 1 & 0 & 0 \\
-1 & 0 & 0 & 0 \\
0 & 0 & 0 & 1 \\
0 & 0 & -1 & 0
\end{array}\right)
$$

\section{The Calabi-Yau threefold case}

Consider a sheaf $V$ and write its Chern character as $\operatorname{ch}(V)=(n, x \sigma+S, \sigma \eta+a F, s)$ $\left(\eta, S \in p_{2}^{*} H^{2}(B)\right)$, then, using the decompostion of the cohomology, we find

$$
\operatorname{ch}(V)=\left(\begin{array}{c}
n \\
x \\
S \\
\eta \\
a \\
s
\end{array}\right), \quad \operatorname{ch}(\hat{S}(V))=\left(\begin{array}{c}
0 \\
-n \\
\eta+\frac{1}{2} x c_{1} \\
-\frac{1}{2} n c_{1}-S \\
s+\frac{1}{2} \eta c_{1} \sigma+\frac{1}{12} x c_{1}^{2} \sigma \\
-\frac{1}{6} n \sigma c_{1}^{2}-a-\frac{1}{2} \sigma c_{1} S+x \sigma c_{1}^{2}
\end{array}\right)
$$

3 The functor $T$ was introduced [4] because its inverse transform in the sense of (13) is the "natural" one: $T$ is the FM transform w.r.t. the sheaf $\overline{\mathcal{P}}=\mathcal{P} \otimes q^{*} \mathcal{O}(F)$ and its inverse functor $\hat{T}$ is the FM transform w.r.t. the dual sheaf $\overline{\mathcal{P}}^{*} . S$ does not have this property as its inverse transform is not the FM transform with respect to $\mathcal{P}^{*}$, but this twisted by $q^{*} K_{B}^{-1}$. So we just divided this up in two parts of $K_{B}^{-1 / 2}$, distributed among the original and the inverse transform. 
If we multiply $\operatorname{ch}(\hat{S}(V))$ by the Todd class $T d(N)=1-\frac{1}{2} c_{1}+\frac{1}{12} c_{1}^{2}$, 1 we get

$$
\operatorname{ch}(\hat{S}(V)) \cdot T d(N)=\left(\begin{array}{c}
x \\
-n \\
\eta \\
-S \\
s-\frac{1}{12} x \sigma c_{1}^{2} \\
-a+x \sigma c_{1}^{2}
\end{array}\right)
$$

When $V$ is fibrewise of degree 0 we have $x=0$ and then

$$
\left(\begin{array}{c}
x \\
-n \\
\eta \\
-S \\
s \\
-a
\end{array}\right)=\left(\begin{array}{cccccc}
0 & 1 & 0 & 0 & 0 & 0 \\
-1 & 0 & 0 & 0 & 0 & 0 \\
0 & 0 & 0 & 1 & 0 & 0 \\
0 & 0 & -1 & 0 & 0 & 0 \\
0 & 0 & 0 & 0 & 0 & 1 \\
0 & 0 & 0 & 0 & -1 & 0
\end{array}\right) \cdot\left(\begin{array}{c}
n \\
x \\
S \\
\eta \\
a \\
s
\end{array}\right)
$$

that is

$$
\operatorname{Td}(N) \cdot \operatorname{ch}(\hat{S}(V))=M \cdot \operatorname{ch}(V) .
$$

So for fibrewise degree 0 , semistable bundles $V$ we have $\hat{S}^{0}(V)=0$ and $\hat{S}^{1}(V)=i_{*} L$ and

$$
\operatorname{Td}(N) \cdot \operatorname{ch}\left(i_{*} L\right)=T d(N) \cdot \operatorname{ch}\left(\hat{S}^{1}(V)\right)=-M \cdot \operatorname{ch}(V) .
$$

The interpretation 6 is that here one must either twist the operation a little bit and stick to the usual Mukai-vector or one keeps the operation and makes the twist in the usual Mukaivector more 'relative'. Explicitly, similarly to the usual twist in the Mukai-vector, here in the fibrewise situation a twist by $\sqrt{\operatorname{Td}(N)}$ with the normal bundle $N=\left(j^{*} T X\right) / T B=$ $j^{*} T_{X / B}=j^{*}\left(T X / \pi^{*} T B\right.$ ) plays a role (where $\left.j: B \hookrightarrow X\right)$. For a complex of sheaves $\mathcal{G}$ on $X$ you twist the standard definition of $c h$ to define a "charge" (where $|i+1|=(-1)^{i+1}$ )

$$
Q(\mathcal{G})=\sum(-1)^{i} \operatorname{ch}\left(\mathcal{G}^{i}\right)(\sqrt{\operatorname{Td}(N)})^{|i+1|}
$$

\footnotetext{
4 We always confuse the normal bundle $N$ with its pull-back $\pi^{*} N$ to $X$

5 There is an analogous equation to (19) for the direct FM transform $S(V)$ : if $x=0$ then $T d\left(N^{-1}\right) \cdot \operatorname{ch}(S(V))=M \cdot \operatorname{ch}(V)$. For fibrewise degree 0 and semistable bundles $V$ we have $S^{0}(V)=$ 0 and with $S^{1}(V)=i_{*} \bar{L}$, we have $T d\left(N^{-1}\right) \cdot \operatorname{ch}\left(i_{*} \bar{L}\right)=\operatorname{Td}\left(N^{-1}\right) \cdot \operatorname{ch}\left(S^{1}(V)\right)=-M \cdot \operatorname{ch}(V)$.

6 In the three-fold case the sole use of the T-functor from the K3-case (for the map between the Chern classes of the bundle and its dual) is insufficient to exhibit (5) as transformation matrix. Rather one needs (just as one has in the usual full, not fibrewise, FM transform actually to take the Mukai vector with its twist by $\sqrt{\operatorname{Td}(X)}$ and not just $\operatorname{ch}(V))$ the relevant twisted charge.
} 
Then

$$
Q(\hat{S}(V))=-\operatorname{ch}\left(\hat{S}^{1}(V)\right)(\sqrt{T d(N)}) \quad, \quad Q(V)=\operatorname{ch}(V)(\sqrt{T d(N)})^{-1}
$$

and thus

$$
Q(\hat{S}(V))=M \cdot Q(V)
$$

Since $\hat{S}^{0}(V)=0$ and $\hat{S}^{1}(V)=i_{*} L$ we have $\hat{S}(V)=i_{*} L[-1]$ as complexes and (21) gives

$$
Q\left(i_{*} L[-1]\right)=M \cdot Q(V)=M \cdot Q\left(S\left(i_{*} L\right)\right)
$$

Alternatively the cohomology effect of the FM transform can be described by a so-called f-map. For a fibrewise FM transform we can also introducd a relative version $\mathbf{f}_{r}: H(X) \rightarrow$ $H(X)$. But modifying $\mathbf{f}_{r}$ to

$$
\mathbf{f}(x)=p_{1 *}\left(p_{2}^{*}(x) \cdot Z\right)
$$

with $Z=\sqrt{p_{2}^{*} \operatorname{Td}(X)} \cdot \operatorname{ch}(\mathcal{P}) \cdot \sqrt{p_{1}^{*} \operatorname{Td}(X)}$, the effective charge of $V$ transforms to

$$
\mathbf{f}(Q(S(V)))=M \cdot Q(V)
$$

when $x=0$. If $V$ is also fibrewise semistable so that $S^{0}(V)=0$ and $S^{1}(V)=i_{*} L$, then

$$
-\mathbf{f}\left(Q\left(i_{*} L\right)\right)=-\mathbf{f}\left(Q\left(S^{1}(V)\right)\right)=\mathbf{f}(Q(S(V)))=M \cdot Q(V)
$$

Fibrewise T-duality on D-branes at the sheaf level

Note that we can also describe the fibrewise T-duality maps in (1) at the sheaf level. Let us consider the skyscraper sheaf $\mathbf{C}(x)$ at a point $x$ of $X$. Its FM transform $S^{0}(\mathbf{C}(x))$

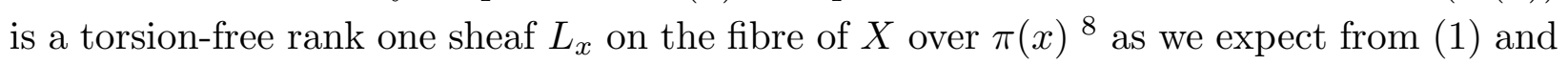
thus $D 0 \rightarrow D 2_{F}$. The topological invariants are $n=x=a=S=\eta=0, s=1$ and

$$
c h_{i}\left(S^{0}(\mathbf{C}(x))\right)=0, \quad i=0,1,3, \quad c h_{2}\left(S^{0}(\mathbf{C}(x))\right)=F
$$

If we start with $\mathcal{F}=\mathcal{O}_{\sigma}$; proceeding as in (3.16) of [0] we have

$$
\begin{aligned}
S^{0}\left(\mathcal{O}_{\sigma}\right) & =\mathcal{O}_{X}, & & S^{1}\left(\mathcal{O}_{\sigma}\right)=0 \\
S^{0}\left(\mathcal{O}_{X}\right) & =0, & & S^{1}\left(\mathcal{O}_{X}\right)=\mathcal{O}_{\sigma} \otimes \pi^{*} K_{B}
\end{aligned}
$$

7 It is defined by $\mathbf{f}_{r}(x)=p_{1 *}\left(p_{2}^{*}(x) \cdot Z_{r}\right)$ where $Z_{r}=\sqrt{p_{2}^{*} T d\left(T_{X / B}\right)} \cdot \operatorname{ch}(\mathcal{P}) \cdot \sqrt{p_{1}^{*} T d\left(T_{X / B}\right)}$. Then $\sqrt{\operatorname{Td}\left(T_{X / B}\right)} \cdot \operatorname{ch}(S(V))=\mathbf{f}_{r}\left(\operatorname{ch}(V) \cdot \sqrt{\operatorname{Td}\left(T_{X / B}\right)}\right)$. The effect of $\mathbf{f}_{r}$ on the effective charge given by the Mukai vector $Q(V)=\operatorname{ch}(V) \cdot \sqrt{T d(X)}$ is described by $\mathbf{f}_{r}(Q(V))=Q(S(V))$.

8 With the identification $X \simeq \tilde{X}$ the point $x$ corresponds precisely to $L_{x}$ (see [4] or [5] ) 
Then $\mathcal{O}_{\sigma}$ transforms to the structure sheaf of $X$ and $\mathcal{O}_{X}$ transforms to a line bundle on

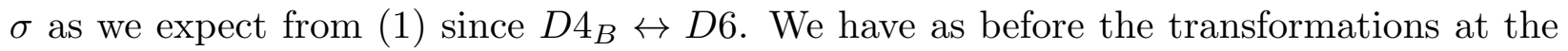
cohomology level $\left(n=0, x=1, S=0, \eta=\frac{1}{2} c_{1}, a=0, s=\frac{1}{6} \sigma c_{1}^{2}\right)$ and get

$$
\operatorname{ch}_{0}\left(S^{0}\left(\mathcal{O}_{\sigma}\right)\right)=1, \quad c h_{i}\left(S^{0}\left(\mathcal{O}_{\sigma}\right)\right)=0, \quad i=1,2,3
$$

Finally, let us consider a sheaf $\mathcal{F}$ on $B$; by (26) we have $S^{0}\left(\mathcal{O}_{\sigma} \otimes \pi^{*} \mathcal{F}\right)=\pi^{*} \mathcal{F}, S^{1}\left(\mathcal{O}_{\sigma} \otimes\right.$ $\left.\pi^{*} \mathcal{F}\right)=0, S^{0}\left(\pi^{*} \mathcal{F}\right)=0 S^{1}\left(\pi^{*} \mathcal{F}\right)=\mathcal{O}_{\sigma} \otimes \pi^{*} \mathcal{F} \otimes \pi^{*} K_{B}$ Then, a sheaf $\mathcal{O}_{\sigma} \otimes \pi^{*} \mathcal{F}=j_{*} \mathcal{F}$ ( $j: B \rightarrow X$ is the section) supported on a curve $\tilde{C}$ in $B$ embedded in $X$ via $j$ transforms to a sheaf on the elliptic surface supported on the inverse image of $\tilde{C}$ in $X$ and vice versa. So we have relations (1) for fibrewise T-duality on D-branes at the sheaf level (cf. [6] )

$$
\begin{aligned}
D 4_{B} & \rightarrow \tilde{D} 6 \\
D 2_{B} & \rightarrow \tilde{D} 4_{\tilde{F}} \\
D 0 & \rightarrow \tilde{D} 2_{\tilde{F}}
\end{aligned}
$$

On the monodromy interpretation of the FM transform

We finally ask whether the Fourier-Mukai transform will be related to a monodromy matrix in the sense of Kontsevich's proposal. We are interested first in an explicit map between the topological invariants of the characteristic classes of the Chan-Paton sheaf $V$ and the brane charges. Consider the BPS charge lattice which can be identified with the middle cohomology lattice of the mirror manifold $H^{3}(\hat{X}, \mathbf{Z})$ and consider (we think of two-parameter models) the central charge associated to the integral vector $\mathbf{n}=\left(n_{6}, n_{4}^{1}, n_{4}^{2}, n_{0}, n_{2}^{1}, n_{2}^{2}\right)$ which is

$$
Z(\mathbf{n})=n_{6} \Pi_{1}+n_{4}^{1} \Pi_{2}+n_{4}^{2} \Pi_{3}+n_{0} \Pi_{4}+n_{2}^{1} \Pi_{5}+n_{2}^{2} \Pi_{6} .
$$

One the other side, one has in the large volume limit of $X$ the lattice of microscopic Dbrane charges (which is identified with the K-theory lattice $K(X)$ ). Here one considers the effective charge $Q$ of a D-brane state $\eta$ given by the Mukai vector $Q=\operatorname{ch}(\eta) \sqrt{\operatorname{Td(X)} \in}$ $H^{\text {even }}(X)$ with the associated central charge

$$
Z(t)=\int_{Z} \frac{t^{3}}{6} Q^{0}-\frac{t^{2}}{2} Q^{2}+t Q^{4}-Q^{6}
$$

where $t$ is the generic Kähler class and one gets

$$
Z(Q)=\frac{r}{6} t^{3}-\frac{1}{2} \operatorname{ch}_{1}(V) t^{2}+\left(\operatorname{ch}_{2}(V)+\frac{r}{24} c_{2}(X)\right) t-\left(\operatorname{ch}_{3}(V)+\frac{1}{24} \operatorname{ch}_{1}(V) \operatorname{ch}_{2}(V)\right)
$$

The comparison of $Z(\mathbf{n})$ and $Z(t)$ leads then to a map between the low energy charges $\mathbf{n}$ and the topological invariants of the K-theory class $\eta$. 
The monodromy transformations $S_{D}$ correspond to automorphisms $M(D)$

$$
[V] \rightarrow\left[V \otimes \mathcal{O}_{X}(L)\right], \quad[V] \rightarrow\left[V \otimes \mathcal{O}_{X}(H)\right]
$$

and we see that the linear transformations acting on $\mathbf{n}$ corresponding to $D$ are

$$
M(D)=S_{D}^{-1}
$$

Let us consider now a second type of monodromy transformation proposed by Kontsevich. He proposed that the monodromy $T$ about the conifold locus of the mirror corresponds to the automorphism of the derived category whose effect on cohomology is

$$
\mathcal{S}: \gamma \rightarrow \gamma-\left(\int \gamma \wedge \operatorname{Td}\left(T_{X}\right)\right) \cdot \mathbf{1}_{X}
$$

$\left(\mathbf{1}_{X}\right.$ the standard generator of $\left.H^{0}(X, \mathbf{Q})\right)$ leading to a change in the topological invariants

$$
\operatorname{ch}(V) \rightarrow \operatorname{ch}(V)-\left(\frac{c h_{1}(V) c_{2}(X)}{12}+\operatorname{ch}_{3}(V)\right)
$$

Using the expression of the prepotential in the large radius limit $\mathcal{F}=\frac{1}{6}(t \cdot J)^{3}-\frac{c_{2}(X)}{24}(t$. $J)+\ldots$ where $(t \cdot J)=\sum t_{a} J^{a}$ and using the period vector $\Pi$ we find the expression valid for both models $\mathbf{P}_{1,1,2,2,2}^{4}[8]$ and $\mathbf{P}_{1,1,2,2,6}^{4}[12]$

$$
\begin{aligned}
& \operatorname{ch}_{1}(V)=n_{4}^{1}\left(J_{1}-2 J_{2}\right)+n_{4}^{2} J_{2} \\
& c h_{3}(V)=-\left(n_{4}^{1}\left(J_{1}-2 J_{2}\right)+n_{4}^{2} J_{2}\right) \frac{c_{2}(X)}{12}-n_{0}
\end{aligned}
$$

leading to the universal shift

$$
n_{6} \rightarrow n_{6}+n_{0}
$$

comparing this to the monodromy we find for the linear transformation acting on $\mathbf{n}$

$$
\mathcal{S}=T^{-1}
$$

We now ask whether the Fourier-Mukai transform will be related to a monodromy matrix in the sense of Kontsevich's proposal. As the corresponding matrices are by mirror symmetry identifiable already on the bundle side this comes down essentially to the question whether the matrix $M$ in (5), is generated by $S_{H}, S_{L}, T$. Recall that the transformations in the Kontsevich association can themselves be considered as FM transforms. This time we will not work on the fibre product but rather on the ordinary product.

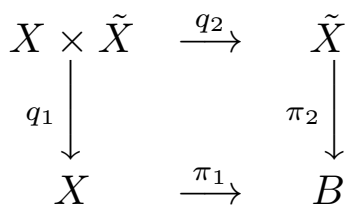


The Fourier-Mukai functors $S^{i}$ are $(\mathcal{E} \in D(X \times X)$ in the derived category)

$$
S_{\mathcal{E}}^{i}(V)=R^{i} p_{1 *}\left(p_{2}^{*}(V) \otimes \mathcal{E}\right)
$$

We can also define the full FM transformation at the derived category level $S_{\mathcal{E}}(\mathcal{G})=$ $R p_{1 *}\left(p_{2}^{*}(\mathcal{G}) \otimes \mathcal{E}\right)$. For example the twist transformation $\mathcal{G} \rightarrow \mathcal{G} \otimes \mathcal{L}$ considered in (32) comes from $\mathcal{E}=\mathcal{O}_{\Delta} \otimes q_{2}^{*}(\mathcal{L})$ where $\Delta$ is the diagonal of $X \times X$. The other operation (corresponding to the 'conifold monodromy') considered before corresponds to an $\mathcal{E}$ whose cohomology is the ideal sheaf $\mathcal{I}_{\Delta}$ of the diagonal of $X \times X$. This recovers the "gamma shift" (34)

$$
\operatorname{ch}\left(S_{\mathcal{I}_{\Delta}}(\mathcal{G})\right)=\operatorname{ch}\left(S_{\mathcal{O}_{X \times X}}(\mathcal{G})\right)-\operatorname{ch}(\mathcal{G})=\left(\int \operatorname{ch}(\mathcal{G}) \wedge \operatorname{Td}\left(T_{X}\right)\right)-\operatorname{ch}(\mathcal{G})
$$

Our fibrewise FM transform is specified by using $\mathcal{E}=j_{*} \mathcal{P}$ where $j: X \times_{B} X \rightarrow X \times X$ is the natural embeddinge ${ }^{2}$, so it is build up out of the divisors (resp. their associated line bundles) $\sigma$ and $\pi^{*} K_{B}$ on the one hand and by $\mathcal{O}_{\Delta}$ on the other.

We have the $M$ matrix equation for the inverse FM transform. This is $\hat{S}=S_{j_{*} \mathcal{Q}}$ with $\mathcal{Q}=\mathcal{P}^{*} \otimes q^{*}\left(K_{B}^{-1}\right)=\mathcal{I} \otimes p_{1}^{*} \mathcal{O}(\sigma) \otimes p_{2}^{*} \mathcal{O}(\sigma) \otimes q^{*} K_{B}^{-2}$ where $\mathcal{I}$ is the ideal of the diagonal of $X \times_{B} X$. Then $\hat{S}=\left(\otimes q^{*} K_{B}^{-2}\right) \circ(\otimes \mathcal{O}(\sigma)) \circ S_{j_{*} \mathcal{I}} \circ(\otimes \mathcal{O}(\sigma))$ or

$$
\hat{S}=S_{\mathcal{O}_{\Delta}\left(2 c_{1}\right)} \circ S_{\mathcal{O}_{\Delta}(\sigma)} \circ S_{j_{*} \mathcal{I}} \circ S_{\mathcal{O}_{\Delta}(\sigma)}
$$

Having written our fibrewise FM transform $\hat{S}$ as a composition of three divisor FM transforms and one FM transform $S_{j_{*} \mathcal{I}}$ we expect the following relation for its monodromy, expressed as a product of divisor monodromies and the monodromy around the diagonal of the fibre product of ideal sheaf $\mathcal{I}$

$$
S_{V}=S_{\sigma} \cdot S_{c_{1}}^{2} \cdot S_{\mathcal{I}} \cdot S_{\sigma}
$$

Let us make this more explicit by considering the model given by the elliptic fibration $\mathbf{P}_{1,1,1,6,9}^{4}[18]$, extensively studied in the context of mirror symmetry [7] and in the context of D-branes on elliptic Calabi-Yau [8]. Among the degree 18 hypersurfaces is 10

$$
z_{1}^{18}+z_{2}^{18}+z_{3}^{18}+z_{4}^{3}+z_{5}^{2}=0
$$

$9 \mathrm{FM}$ on $X \times_{B} X$ with respect to $P$ is FM on $X \times X$ with respect to $j_{*} P$

10 At $z_{1}=z_{2}=z_{3}=0$ the ambient space has a singular line which intersects $X$ in a single point. The blow up of this line gives an exceptional divisor $E=P^{2}$ in $X$. A second divisor $L$ (defined by the first order polynomials) is given by the elliptic surface over a line in $P^{2}$ and together with $E$ generates $H_{4}(X, \mathbf{Z})$. The elliptic fibration structure is induced by the linear system $|L|$ generated by $z_{1}, z_{2}, z_{3}$ mapping $X$ to $\mathbf{P}^{2}$. The section of the fibration is given by $B_{2}=E$. The homology class of the elliptic fibre in $H_{2}(X)$ will be denoted by $h=L^{2}$. Further intersection relations are given by $E \cdot L^{2}=1, \quad E^{2} \cdot L=-3, \quad L^{3}=0, \quad E^{3}=9$. Working in the $E, L$ basis (cf. [8]) the generic Kähler class is given by $J=t_{1} E+t_{2} L$ with $t_{1}, t_{2}$ coordinates on the Kähler moduli space. In the degree 18 model again one has $\sigma=E=B=P^{2}$ and $\pi^{*} K_{B}=3 L$; note that $H=3 L+E$ with a corresponding multiplicative relation $S_{E}=S_{H} \cdot S_{L}^{-3}$ for the matrices. 
Here the relation becomes

$$
S_{V}=S_{E} \cdot S_{L}^{6} \cdot S_{\mathcal{I}} \cdot S_{E}
$$

where the commuting (cf. [9], p. 12) matrices $S_{L}, S_{E}$ are given by

$$
S_{L}=\left(\begin{array}{cccccc}
1 & 0 & -1 & 3 & 2 & 0 \\
0 & 1 & 0 & 1 & 0 & -1 \\
0 & 0 & 1 & 0 & -1 & 0 \\
0 & 0 & 0 & 1 & 0 & 0 \\
0 & 0 & 0 & 0 & 1 & 0 \\
0 & 0 & 0 & 1 & 0 & 1
\end{array}\right), \quad S_{E}=\left(\begin{array}{cccccc}
1 & -1 & 0 & 1 & 0 & 0 \\
0 & 1 & 0 & -9 & 0 & 3 \\
0 & 0 & 1 & 3 & 0 & -1 \\
0 & 0 & 0 & 1 & 0 & 0 \\
0 & 0 & 0 & 1 & 1 & 0 \\
0 & 0 & 0 & -3 & 0 & 1
\end{array}\right)
$$

In order to obtain the matrices $S_{\mathcal{I}}$ and $S_{V}$ we have to use the comparison (performed for this model in [8]) of the central charges $Z(\mathbf{n})$ and $Z(Q)$ which gives the relation between the middle cohomology charges $\mathbf{n}$ and the cohomological invariants of the vector bundle $\mathcal{G}$

$$
\operatorname{ch}(\mathcal{G})=\left(n, \alpha E+\beta L, \gamma E L+\delta L^{2}, \epsilon\right)
$$

where the coefficients are given in terms of the BPS charge vector $\mathbf{n}$

$$
(n, \alpha, \beta, \gamma, \delta, \epsilon)=\left(n_{6}, n_{4}^{1}, n_{4}^{2}, \frac{3}{2} n_{4}^{1}+n_{2}^{2}, \frac{3}{2} n_{4}^{2}+n_{2}^{1},-n_{0}+\frac{1}{2} n_{4}^{1}-3 n_{4}^{2}\right)
$$

The FM transforms $\hat{S}($.$) and S_{\mathcal{I}}($.$) induce a linear transformation on the BPS charge lattice$

$$
S_{\mathcal{I}}=\left(\begin{array}{cccccc}
1 & 0 & 3 & -9 & 0 & 0 \\
1 & 1 & 3 & -9 & -1 & 0 \\
0 & 0 & 1 & 0 & 0 & 0 \\
0 & 0 & 0 & 1 & 1 & 0 \\
0 & 0 & 0 & 0 & 1 & 0 \\
0 & 0 & 1 & -3 & 0 & 1
\end{array}\right), \quad S_{V}=\left(\begin{array}{cccccc}
0 & -1 & 0 & 1 & 0 & 3 \\
1 & 0 & 0 & 0 & -1 & 0 \\
0 & 0 & 0 & 0 & -3 & -1 \\
0 & 0 & 0 & 0 & -1 & 0 \\
0 & 0 & 0 & 1 & 0 & 0 \\
0 & 0 & 1 & -3 & -3 & 0
\end{array}\right)
$$

with $S_{\mathcal{I}}^{-1}=P\left(S_{\mathcal{I}}(\cdot)\right), S_{V}^{-1}=P(\hat{S}(\cdot))$ denoting the linear transformations on the lattice $\mathbf{n}$.

Finally, using these matrices we can write the $M$ matrix as (for $\alpha=n_{4}^{1}=0 ; l$ relates (cf. (47)) the period basis with the 'fibration' basis (21), $S_{t d}$ represents the $T d(N)$ twist )

$$
M=l \cdot\left[S_{V}^{-1}\right]^{t} \cdot l^{-1} \cdot S_{t d}
$$

Let us close with some remarks:

1) First we want to point to some analogues on the mirror side. Note that (48) also shows that if we perform a linear transformation on $H^{3}(Y)$ (transforming in the "fibre base") by $l$ the $M$ matrix naturally operates on the charge lattice as

$$
\left(\begin{array}{l}
n \\
\alpha \\
\beta \\
\gamma \\
\delta \\
\epsilon
\end{array}\right)=M \cdot\left(\begin{array}{c}
-\alpha \\
n \\
-\gamma \\
\beta \\
-\epsilon \\
\delta
\end{array}\right)
$$


and thus (48) gives just the right transport of the $M$ matrix to $H^{3}(Y)$

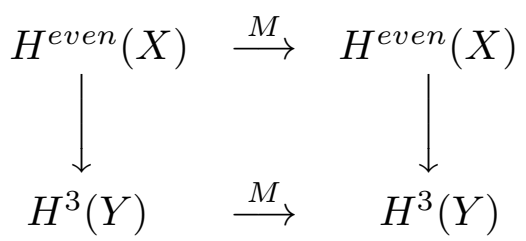

Thus the $l$ transformation shows how to get the 'fibration' basis (2) from the Kähler period vector basis, resp. (after identification with the mirror side) how the (2) basis (when transported via identification to the mirror side) is related to the complex structure period vector basis, making explicit the decomposition in $H^{3}(Y)$ corresponding to (2)

$$
H^{3}(Y)=H^{\text {non-ell }} \oplus H^{e l l}
$$

One can ask whether this decomposition and 'duality' transformation on the middle cohomology of $Y$ comes actually from a natural decomposition and map on the space $Y$. Also, in the context of the extended mirror conjecture [10] with its $h^{1}(\operatorname{End}(V), X)=h^{1}(C)$, one can transport detailed information about $V$ from index computations.

2) For the line bundle $L$ on the spectral cover $C$ one has

$$
0 \rightarrow H^{1}\left(C, \mathcal{O}_{C}\right) \rightarrow \operatorname{Ext}_{X}^{1}\left(i_{*} L, i_{*} L\right) \rightarrow H^{0}\left(C, \mathcal{O}_{C}\right) \rightarrow 0
$$

so that

$$
\begin{aligned}
\operatorname{dim} \operatorname{Ext}_{D}^{1}(L, L) & =h^{1}\left(C, \mathcal{O}_{D}\right)=h^{(0,1)}(C) \\
\operatorname{dim} \operatorname{Ext}_{X}^{1}\left(i_{*} L, i_{*} L\right) & =h^{1}\left(C, \mathcal{O}_{D}\right)+h^{0}(C, N)=h^{(0,1)}(C)+h^{(2,0)}(C)
\end{aligned}
$$

The vector bundle $V=S^{0}\left(i_{*} L\right)$ has as unique inverse $\mathrm{FM}$ transform $\hat{S}^{1}(V)=i_{*} L$,so

$$
\operatorname{Ext}_{X}^{1}(V, V)=\operatorname{Ext}_{X}^{1}\left(\hat{S}^{1}(V), \hat{S}^{1}(V)\right)=\operatorname{Ext}_{X}^{1}\left(i_{*} L, i_{*} L\right)
$$

(by the "Parceval isomorphism") and then

$$
\begin{aligned}
& \operatorname{dim} \operatorname{Ext}_{D}^{1}(L, L)=h^{1}\left(C, \mathcal{O}_{D}\right)=h^{(0,1)}(C) \\
& \operatorname{dim} \operatorname{Ext}_{X}^{1}(V, V)=h^{1}\left(C, \mathcal{O}_{D}\right)+h^{0}(C, N)=h^{(0,1)}(C)+h^{(2,0)}(C)
\end{aligned}
$$

Actually one can see explicitly that $\operatorname{dim} E x t_{X}^{1}(V, V)=\operatorname{dim} E x t_{X}^{1}\left(i_{*} L, i_{*} L\right)$ without using (50) : use the character valued index and compute in the spectral cover representation (cf. [11] ) the relation $\operatorname{dim} H^{1}(X, \operatorname{End}(V))=h^{(2,0)}(C)+h^{(1,0)}(C)$ showing the agreement. 


\section{References}

[1] B. Andreas, G. Curio, D. Hernández Ruipérez and S.-T. Yau, "Fourier-Mukai Transform and Mirror Symmetry for D-Branes and Elliptic Calabi-Yau", math. AG/0012196.

[2] R. Friedman, J. Morgan and E. Witten, "Vector Bundles and F- Theory," Commun. Math. Phys. 187 (1997) 679, hep-th/9701162.

[3] T. Bridgeland, "Fourier-Mukai transforms for elliptic surfaces", J. reine angew. Math. 498, alg-geom/9705002.

[4] C. Bartocci, U. Bruzzo, D. Hernández Ruipérez and J. M. Muñoz Porras, "Mirror symmetry on K3 surfaces via Fourier-Mukai transform", Commun. Math. Phys. 195 (1998), 79-93

[5] D. Hernández Ruipérez and J. M. Muñoz Porras, "Structure of the moduli space of stable sheaves on elliptic fibrations", math. AG/9809019.

[6] R. Donagi, B. Ovrut, T. Pantev and D. Waldram, "Standard Models from Heterotic M-theory", hep-th/9912208.

[7] P. Candelas, A. Font, S. Katz and D. Morrison, "Mirror Symmetry for Two Parameter Models- II", hep-th/9403187.

[8] D.-E. Diaconescu and C. Römelsberger, "D-Branes and Bundles on Elliptic Fibrations", hep-th/9910172.

[9] P. Candelas, X. De La Ossa, A. Font, S. Katz and D. R. Morrison, "Mirror symmetry for two parameter models I", Nucl. Phys. B416 (1994) 481, hep-th/9308083.

[10] C. Vafa, "Extending Mirror Conjecture to Calabi-Yau with Bundles", hep-th/9804131.

[11] G. Curio and R. Donagi, "Moduli in N=1 Heterotic/F-Theory Duality", Nucl.Phys. B518 (1998) 603, hep-th/9801057. 